Zagreb

\title{
ETRUSCAN NUMERALS
}

The origin of the Etruscan numerals was tried to be explained in many ways, considering the meanings given to numerals. In this article etymologies of the Etruscan numerals are given with regard to the order given by Wilkins, Pfiffig and Izbicki - vu, zal, ci, śa, max, huv, sem $\varphi$, cezp, nurq, śs. 1

Yu "one" came from

Nostr. *'UXdE or * 'UQdE "one, only":

HS * 'UHdh: Sem. * 'hd "one": Akkad. èdu "individual, solitary, single", Hebr. "ehād "one", Arabic "ahad- id.; Sem. *whd "one, single": Akkad. wēdum "one", Hebr. jāhīd id., Arabic wahịd "single"; Eg. $\underline{w}^{c}$ m., w․t f. "one" $<$ HS *WAHd-;

Kart. *ode "until": Georg. oden "when", odes id., -ode "only" (oriode "only two", semiode "only three"), Megrel. odo- "until", Svan wode id.;

IE *ed(h)-: Sl. *edbn-/edinb "one": OSl. jedinz, jedino/jedbno, Russ. odín, odnó, SCr. jèdan etc.; *ede: OSl. jede koi "some";

FU *ükte "one": Finn. yksi, yhte-, SaamiN oktâ, Erzyan vejke, Mokshan if ${ }^{\prime k \varepsilon}$, Mari ik, Udmurtian odik, Komi et, Hung. égy etc.;

Dr. *ut- "what forms a pair": Telugu uddi "match, equal, rival", Tulu udri "match, pair" etc.;

Alt.: Tung. *ragda "only": Nanai ogdadi-ragda "in boats only", Evenki bäje-riktä-1 "men only"; *-r- is Tungus suffix of cardinal numbers; 2

Yeniseian *qūd/t "one": Ket kūs, qùs, Imbat xusam, Sym uuse, kūs etc., Pumpokol' xuta. 3

zal, esal "two" came from

Nostr. *ĆoLfa "to be a pair, to mate":

HS *c^l - "to be a pair": Arabic sal' ${ }^{c}$, sil" - "semblable, like, fellow"; Southern Cushitic (Iraqwi) *cat- $\left(<{ }^{*}\right.$ cat- $\left.<{ }^{*}{ }^{* \Lambda l^{k}}-\right)$ : Iraqw, Gorowa, Alagwa

1 Pfiffig 1969:123 ff.

2 Dolgopol'skij 1969:300-1, HS+Kart.+IE+FU+Dr.+Tung.

3 Toporov 1967:315 


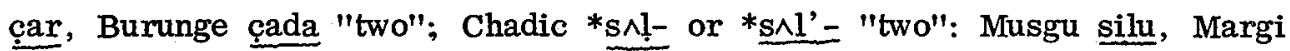
sàdà, Logone xsda etc.;

Kart. *Cgwil- "pair": Georg. çqwil-i "pair, testicles";

Ural. *sóf'a- "to mate, to aspire for mating": Finn. suota "group of mares during a time of mating", Nenets sāje- "to mate (of birds)"; 4

ci "three"

The connection with Urartean kig? "three" in kigarbu "threeyear" is possible. 5

śa "four"

Taking into consideration the connection between "four" and "six" (f.e., IE * $\mathrm{k}^{\mathrm{W}}$ etwer- "four", FU *kutte "six", Mong. *ketüre "to be to many/much, exceed the norm", Tung. *kätö- "much, many"), 6 we can compare Etr. śa "four" with

Kart. *eksw- "six": Georg. ekws-, Megrel. amšw-, Chan anš-, Svan usgwa-, uskiwa-; 7

IE *(s)weks/uḱs "six": OInd. șaș-, Gr. héx, Lat. sex, Goth. saihs, Lith.

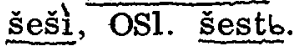

Still Fr. Bopp thought about this connection between Kartvelian and IndoEuropean name of "six", thinking Georgian is Indo-European language. 8

G. A. Klimov thinks that the Kartvelian word is the borrowing from IndoEuropean. 9

Very interesting material is found in Vietnamuong: Vietnamese sáu, Muong kháu (Proto-Vietnamuong * [̌s ] aw "six"). 10

max "five" can be explained by

Nostr. *m^gE "much, many, big":

HS: Cush. *mngg- (/mng-?) "full, to fill": Afar mag- "to fill", Somali mug "lots, stoutness"; Baiso migi "full"; 11

4 Dolgopol'skij 1972:362, HS+Kart.+Ural.

5 D' jakonov 1967:141

6 Dolgopol'skij 1970:626

7 Klimov 1964:80

8 Bopp 1848:38

9 Klimov 1967:308

10 Barker-Barker 1970:284

11 Dolgopol'skij 1973:256-7 
IE *meǵ(h)- "big": OInd. mahắn, Av. mazănt, Hitt. mekki- "numerous", Gr. mégas "big", Lat. magnus, Goth. mikils; 12

CK *mk "many": Chukchi na-mka-qin, 13 Alyutor na-mka-'a: 14

Niv]kh mxo "ten"15 (compare Nenets jun" Hanti uget, Hung. öt "five"). 16

The link between "much, many" and "full" is clear; for the development "full" $\rightarrow$ "complete" $\rightarrow$ "ten" compare SCr. pưno "much, many": pưn "full" and Eskimo qula "ten" and "top, upper parts; upper" (Eskimos lift both their hands when enumerate ten); 17 "five" is "full hand", "all fingers". Also compare Chukchi ralg-ak "to count": ralgalgan "finger", matlanen "five": mangatlayon "hand".18

huvh "six"

With regard to the connection between the numerals for "four" and "six" (see above, s.v. śa), huh can be compared with

Kart. *otx- "four": Georg. otx-, Megrel. otx-, Chan o(n)txo-, otxu-, Svan wošt $x(w)-19$ and

IE *olktōo(u) "eight": OInd. astāú, Gr. óktō, Lat. octo, Goth. ahtau, Lith. aštuoni, OSl. osmb, where *okto(u) is the dual form of *olkto "four".

G. A. Klimov compares these Indo-European and Kartvelian numerals and explains them as areal parallelism. 20

semp "seven" can be joined to

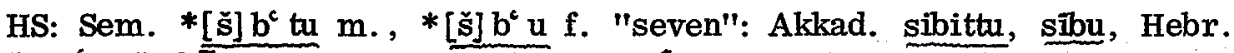

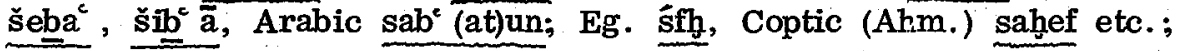

Kart. *šwid- "seven": Georg. šwid-, Megrel. škwit-, Chan šk(w)it-, Svan išgwid-. 21 According to Klimov, *气ّwid- is Semitic borrowing; 22

12 WP II 257-8

13 Skorik 1968:266

14 Žukova 1968:306

15 Panfilov 1968:413

16 Čop 1973:147-8

17 Menovščikov 1962:288-9

18 Skorik 1968:259

19 Klimov 1964:150

20 Klimov 1977:162-3

21 Klimov 1964:216-7

22 Klimov 1967:308 
IE *septm "seven": OInd. saptá, Gr. heptá, Lat. septem, Goth. sibun, Lith. septyni, OS1. sedmb. 23

This word can be the Mediterranean word; compare also Hurrian šitta, šinda "seven". 24

cezp "eight"

can be explained as *ce=ci "three" $+*_{\text {zp }}$, what came from Nostr. *capa, the variant of

Nostr. * japa "to take in hands, to grasp, to gather":

IE *sep- "to take in hands, to be occupied": OInd. sápati "is occupied", Av. hap- "to take in hands", Gr. -epō, aor. -espon "I prepare", maybe S1. *xapati, *ščepati, *capati; 25

Alt. * ̌̌apa- "to take in hands, to work": Turkic: Old Uighur jap- "to settle", Turkish yap- "to do, to work"; Tung. *3apa- "to take, to seize": Manchu ¿afa-, Nanai japao-, Evenki ¿awa-; Korean čab- "to take in hands".26

The Etruscan *zp possibly had the meaning "five" — "hand" (compare Turkish bes, Chuvash pillak "five": Azerbaijani, Uighur biläk "hand") — "to grasp, to gather" (compare OSl. bb rati, bero "to take, to grasp, to gather", OSl. roka, Lith. rankà "hand": Lith. riñkti "to gather").

$\underline{\text { nurep "nine" }}$

can be analysed as *nu "new" + * ${ }^{\text {rop }}$ "eight", where *nu came from

Nostr. *Nüqn "now":

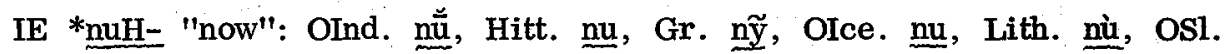
nyné; also *new $(\mathrm{H})-0-$, *neu(H)-jo- "new", what appears in IE *newn "nine" too,

Ural. *Nük^ "now": Finn. nyky- "present", nyt, nyy "now", Mokshan ńi "already", Erzyan ńej "now";27

Eskimo *nu- "new, young": nutaq "new", nukaq "younger brother". 28

The element * $\underline{\underline{r}} \boldsymbol{\varphi}$ means "eight" and it is connected with

Sem.: Arabic arba', Akkad. arba' u "four",

23 see also Illič-Svityč 1964:6, IE+Sem.+Kart.

24 Klimov 1971:53

25 E்SSJa 3.170-1, s.v. *capati

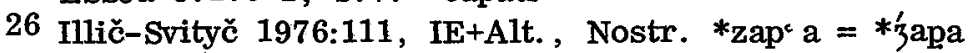

27 Illič-Svityč 1976:97, IE+Ural.

28 Menovščikov 1974:58, IE+Esk., with lit. 
Kart. *arwa- "eight": Georg. rwa-, Megrel. bruo, Chan (")ow ro-, Svan ara-, 29 what is the Semitic borrowing. 30 About "eight": "four" compare IE *oktōo(u) "eight" = "twice four".

So nurq "nine" means "new after eight" (compare IE *newn "nine" < *neu"new").

sar "ten" is connected with

Sem.: Akkad. esertu, Arabic ašr "ten", ašir "tenth",

Kart. *aŝs- "hundred": Georg. as-, Megrel. oš-, Chan oš-, Svan ašir-, äšir-; 31

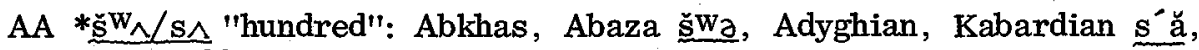
Ubykh $\stackrel{\text { šwa; }}{ } 32$

ND "hundred": Lak tturš, Dargwa darš, Tabassaran warž, Aghul wärš.

G.A. Klimov explains Caucasian words as Semitic borrowings and compares the semantical relation with Manchu juvan "ten" and Mongolian jagun $<$ *žavun "hundred". 33

Also Turkic parallels can be joined here:

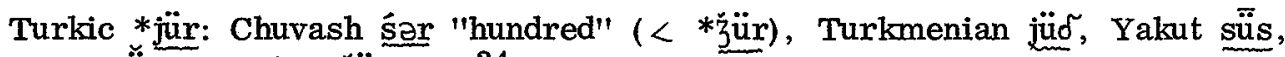

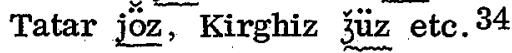

\section{REFERENCES}

Barker, M.A., Barker, M.E. (1970): Proto-Vietnamuong (Annamuong) final consonants and vowels. Lingua 24, 268-85.

Bopp, F. (1848): Die kaukasischen Glieder des indoeuropäischen Sprachstamms. Berlin.

Čop, B. (1973): Indouralica IV. Linguistica XIII, 116-190.

D’jakonov, I.M. (1967): Jazyki drevnej Perednej Azii. Moskva, Nauka.

Dolgopol'skij, A.B. (1969): Nostratičeskie osnovy s sočetaniem šumnyh soglasnyh. Étimologija 1967, 296-313.

\section{Klimov 1964:44-5}

30 see Klimov 1967:308-9

31 Klimov 1964:45, Kart.+AA+ND

32 Džanašia 1976:145 ff., AA+Kart.

33 Klimov 1967:309-10

34 Levitskaja 1976:44 
---, (1970): A long-range comparison of some languages of Northern Eurasia. Problems of phonetic correspondences. VII Meždunarodnyj kongress antropologičeskih i ètnografičeskih nauk. Moskva 3-10 avgusta 1964 g. , Tom V, 620-628. Moskva, Nauka GRVL.

---, (1972): Nostratičeskie korni s sočetaniem lateral' nogo i zvonkogo laringala. Étimologija 1970, 356-69.

---, (1973): Sravnitel'no-istoričeskaja fonetika kušitskih jazykov. Moskva, Nauka.

Džanašia, R.S. (1976): Imena čislitel'nye v abhazsko-adygskih jazykah. Tberiul-kavkasiuri enatmecnierebis celiçdeuli (Ežegodnik iberijsko-kavkazskogo jazykoznanija) III, 144-63.

ĖSSJa 3: Étimologičeskij slovaŕ slavjanskih jazykov. Praslavjanskij leksičeskij fond. Vyp. 3 (*bratr c - *cbrky). Pod red. O.N. Trubačeva. Moskva, Nauka.

Illič-Svityč, V.M. (1964): Drevnejšie indoevropejsko-semitskie jazykovye kontakty. Problemy indoevropejskogo jazykoznanija, 3-12. Moskva, Nauka.

---, (1976): Opyt sravnenija nostratičeskih jazykov. Sravnitel'nyj slovaŕ /ㅍ/ $\left(1-\frac{1}{3}\right)$. Moskva, Nauka.

Klimov, G.A. (1964): Ėtimologičeskij slovaŕ kartvel'skih jazykov. Moskva, Nauka.

---, (1967): Zaimstvovannye čislitel'nye v obščekartvel'skom? Ėtimologija 1965, 307-11.

---, (1971): Voprosy metodiki sravnitel'no-genetičeskih issledovanij. Moskva, Nauka.

---, (1977): Kartvel' skoe *otxo- "četyre" indoevropejskoe *okto-. . Ėtimologija 1975, 162-3.

Levitskaja, L.S. (1976): Istoričeskaja morfologija čuvašskogo jazyka. Moskva, Nauka GRVL.

Menovščikov, G.A. (1962): Grammatika jazyka aziatskih èskimosov I. Moskva-Leningrad, Izdatel'stvo Akademii nauk SSSR.

---, (1974): Ėskimossko-aleutskie jazyki i ih otnošenie $\mathrm{k}$ drugim jazykovym sem' jam. Voprosy jazykoznanija 1/1974, 46-59.

Panfilov, V.Z. (1968): Nivhskij jazyk. Jazyki narodov SSSR V, 408-34. Leningrad, Nauka LO.

Pfiffig, A.J. (1969): Die etruskische Sprache. Versuch einer Gesamtdarstellung. Graz, Akademische Druck- u. Verlagsanstalt. 
Skorik, P.Ja. (1968): Čukotskij jazyk. Jazyki narodov SSSR V, 248-70. Leningrad, Nauka LO.

Toporov, V.N. (1967): Iz ètimologii enisejskih jazykov (K voprosu ob odnom rjade sootvetstvij pumpokol'skomu t). Etimologija 1965, 311-20.

Žukova, A.N. (1968): Aljutorskij jazyk. Jazyki narodov SSSR V, 294-309. Leningrad, Nauka LO.

Sažetak

\section{ETRURSKI BROJEVI}

Porijeklo etrurskih brojeva objašnjavalo se na mnogo načina, ovisno o vrijednostima koje su im davane. Brojevi u poretku Wilkinsa, Pfiffiga i Izbickog daju se etimološki ovako protumačiti:

ऽu "jedan" nastalo je od nostr. * 'UXdE ili * 'UQdE "jedan, sam" (semham. * "UḤd^ "jedan", kart. *ode "samo", ie. *ed(h)- "jedan", uf. *ükte isto, dr. *ut- "što čini par", tung. *ragda "samo", jenisej. *qūd/t "jedan"); zal, esal "dva" nastalo je od nostr. *ĆoL「a "biti par, pariti (se)" (semham. $*_{c \wedge} l^{*}$ - "biti par", kart. *C̣qwil- "par", ural. *sós $\delta^{\prime}$ a- "pariti se, stremiti parenju");

ci "tri" možda je povezano s urart. kig? "tri";

śa "četiri", zbog veze izmedju "četiri" i "šest", može se usporediti s kart. *ekŝw- "šest", ie. *(s) weḱs/uks isto, a zanimljivo je vijetnamuong $*[\mathrm{~s}]$ aw "šest";

max "pet" može se objasniti pomoću nostr. *m^gE "mnogo, velik" (kuš. *m^gg- "pun, puniti", ie. *meǵ(h)- "velik", čukkamč. *mk "mnogo", nivh. mxo "deset"); za semantički razvoj usporedi hs. pưno: pưn: eskim. qul'a "deset" i "vrh, gornji dio, gore";

hu v "šest" da se usporediti s kart. *otx- "četiri", ie. oḱtō(u) "osam" (< *oḱto "četiri");

sem $\varphi$ "sedam" može se dodati $\mathrm{k}$ sem. $*[\check{s}] \mathrm{b}^{\mathrm{c}}$ tu $\mathrm{m}$. "sedam", egip. śfh isto, kart. *šwid- isto, ie. *septm isto; riječ može biti mediteranska, za što usporedi hur. šitta, šinda "sedam";

cezp "osam" može se objasniti kao ce=ci "tri" + *zp što se razvilo od nostr. *éapa, a što je varijanta osnove *zapa "uzeti u ruke, uhvatiti, skupljati" (ie. *sep- "uzeti u ruke, baviti se", alt. *ǰapa- "uzeti u ruke, raditi"); za semantički razvoj usporedi čuvaš. pillək "pet" prema azerb., ujgur. biläk "ruka" te lit. rankà "ruka" prema riñkti "skupljati"; 
nurp "devet" može se analizirati kao *nu "nov" (od nostr. *Nüq^ "sada": ie. *nuH- isto, ural. *Nük^ isto, esk. *nu- "nov, mlad") + *r $\varphi$ "*osam" povezano sa semit.: arap. arba" itd. "četiri", kart. *arwa- "osam" pa nurp znači "novo poslije osam" kao što je ie. *newn "devet" < *neu- "nov"; śar "deset" povezano je sa semit. akad. esertu itd. "deset", kart. *aŝ"sto", abhadig. $*{ }^{\mathrm{s}} \mathrm{w}_{\wedge} / \mathrm{s} \wedge$ isto, nahdag. lak. tturš itd. isto te turk. *jür isto. 